Research Article

\title{
Photophysical Behavior of Modified Xanthenic Dyes Embedded into Silsesquioxane Hybrid Films: Application in Photooxidation of Organic Molecules
}

\author{
Carolina V. Waiman, ${ }^{1,2}$ Rodrigo E. Palacios, ${ }^{2,3}$ Hernán A. Montejano, ${ }^{2,3}$ \\ Carlos A. Chesta, ${ }^{2,3}$ and María Lorena Gómez ${ }^{2,3}$ \\ ${ }^{1}$ Departamento de Química, INQUISUR, Universidad Nacional del Sur (UNS), CONICET, Av. Alem 1253, \\ 8000 Bahía Blanca, Argentina \\ ${ }^{2}$ Consejo Nacional de Investigaciones Científicas y Técnicas (CONICET), Godoy Cruz 2290, C1425FQB CABA, Argentina \\ ${ }^{3}$ Departamento de Química, Universidad Nacional de Río Cuarto, Campus Universitario, 5800 Río Cuarto, Argentina
}

Correspondence should be addressed to María Lorena Gómez; mlgomez@exa.unrc.edu.ar

Received 30 April 2017; Revised 27 June 2017; Accepted 16 July 2017; Published 16 August 2017

Academic Editor: Leonardo Palmisano

Copyright (c) 2017 Carolina V. Waiman et al. This is an open access article distributed under the Creative Commons Attribution License, which permits unrestricted use, distribution, and reproduction in any medium, provided the original work is properly cited.

\begin{abstract}
Polymeric materials based on a bridged silsesquioxane with pendant dodecyl chains were synthesized and modified with different xanthenic dyes with the aim of developing a material with potential application in photooxidation of organic compounds. The employed dyes constitute a family of novel xanthenic chromophores with outstanding properties as singlet oxygen photosensitizers. The hybrid matrix was chosen for its enhanced properties such as flexibility and chemical resistance. The employed dyes were easily incorporated into the hybrid polymer obtaining homogeneous, transparent, and low-refractive-index materials. The polymeric films were characterized using UV-Vis absorption, fluorescence, and laser flash photolysis techniques. The ability of these materials to produce singlet oxygen was tested following the photooxidation of 9,10-dimethylanthracene which is a well-known chemical trap for singlet oxygen. High photooxidation efficiencies were observed for these materials, which present the advantage of being easily removed/collected from the solution where photooxidation takes place. While photobleaching of the incorporated dyes is commonly observed in the solution, it takes place very slowly when dyes are embedded in the hybrid matrix. These properties bode well for the potential use of these materials in novel wastewater purification strategies.
\end{abstract}

\section{Introduction}

Water-soluble nonbiodegradable organic compounds such as herbicides, industrial dyes, and metabolites of massively used therapeutic drugs are some of the main sources of contamination in subterranean water reservoirs of agricultural regions and in domestic and industrial effluents $[1,2]$. Chemical reactions catalyzed by $\mathrm{UV}-\mathrm{V}$ is radiation of organic and inorganic sensitizers are one of the most economically viable methods for decomposing contaminants into innocuous and/or biodegradable subcomponents [3-5]. In particular, nano/microparticulate systems able to form stable aqueous suspensions are highly desirable due to its convenient application and efficient decontamination action in large volumes of fluids (associated with their large specific surface area) $[6,7]$. However, these systems present problems associated with their removal from the treated fluid once its decontamination action has taken place.

Decontamination systems with high chemical stability and the capability of being easily and effectively removed from the treated fluids are highly desirable for reusable and residueless applications $[8,9]$. Construction of such systems requires the development of materials with moderate-tohigh mechanical strength and chemical stability; hybrid materials based on silsesquioxanes represent a family of compounds that can readily fulfill these requirements $[10,11]$. 
<smiles>O=C1OC2(c3cc(I)c(O)c(I)c3Oc3c2cc(I)c(O)c3I)c2c(Cl)c(Cl)c(Cl)c(Cl)c21</smiles>

$\mathrm{RB}$<smiles>CC(=O)c1c(Cl)c(Cl)c(Cl)c(Cl)c1C1c2cc(I)c(O)c(I)c2Oc2c1cc(I)c(O)c2I</smiles>

RB3<smiles>O=C(O)c1ccccc1-c1c2cc(Br)c(=O)c(Br)c-2oc2c(Br)c(O)c(Br)cc12</smiles>

EoY<smiles>CC(=O)c1ccccc1-c1c2cc(Br)c(=O)c(Br)c-2oc2c(Br)c(O)ccc12</smiles>

Eo2<smiles>O=C(O)c1ccccc1-c1c2cc([N+](=O)[O-])c(=O)c(Br)c-2oc2c(Br)c(O)c([N+](=O)[O-])cc12</smiles>

EoB<smiles>CC(=O)c1ccccc1-c1c2cc(Br)c(=O)c(Br)c-2oc2c(Br)c(O)c(Br)cc12</smiles>

Eo3

FIGURE 1: Chemical structure of the studied xanthenic dyes.

Bridged silsesquioxanes (SSO) are a family of organicinorganic hybrid materials obtained via sol-gel from the hydrolysis and polycondensation of monomers having urea groups with an organic bridge group between two (or more) trialkoxysilanes. These materials are attractive for several reasons: they show intrinsic luminescence, are easy to synthesize, and have high chemical, mechanical, and thermal resistance [12-15]. Furthermore, because SSO are porous and/or mesoporous materials, they can be used for homogeneously dispersing within its matrix's diverse kinds of compounds producing materials with new properties for interesting applications [9, 14-16].

On the other hand, the photochemical properties of various xanthenic dyes, mainly rose bengal (RB) and eosin (Eo), have been extensively studied due to their diverse applications that derive mainly from their large molar absorption coefficients in the visible region and their high quantum yields of singlet oxygen generation.

There have been many studies related to the photochemical behavior of xanthenic dyes and structurally related compounds. Since the pioneering work of Gorman and Rodgers [17] and Schaap et al. [18] in the description of singlet oxygen generation photosensitized by xanthene dyes, many researchers have reviewed the subject for new applications of these dyes, being a topic of current interest [19-26].

Herein, we describe the synthesis of polymeric hybrid films with application to the visible light-induced degradation of polyaromatic hydrocarbons, namely, a film system composed of a silsesquioxane matrix with pendant dodecyl chains doped with modified xanthenic dyes (Figure 1) which acts as singlet oxygen $\left({ }^{1} \mathrm{O}_{2}\right)$ photosensitizer.

The synthesis of dye-doped silsesquioxane films was performed using a hydrolysis polycondensation method [12].
The resulting films have uniform optical absorption indicating a homogeneous distribution of the sensitizers in the film. The photophysical properties of the dyes in the films were studied and compared with those in the homogeneous solution. The photostability of the dyes incorporated into the hybrid films is enhanced as compared to that in the solution. These systems are able to sensitize appreciable photooxidation of 9,10-dimethylanthracene (DMA), a well-known chemical trap for singlet oxygen [27], in acetonitrile under visible illumination. In view of these results, the developed films could be attractive systems for applications that require not only efficient photosensitized oxidation capacity but also fast and convenient removal of the sensitizer system from contaminated fluids after treatment.

\section{Materials and Methods}

2.1. Materials. Dodecylamine (DA, 98\%, Fluka), glycidoxypropyltrimethoxysilane (GPTMS, 97\%, Sigma-Aldrich Argentina), formic acid (88 wt\%, Cicarelli), water (HPLC grade, Sintorgan), dimethylsulfoxide (DMSO, HPLC grade, Sintorgan), acetonitrile (ACN, HPLC grade, Sintorgan), and 9,10-dimethylanthracene (DMA, 99\%, Sigma-Aldrich) were used as received. Tetrahydrofuran (THF) (HPLC grade, Cicarelli) was refluxed for 5 hours with potassium hydroxide pellets ( $\mathrm{KOH}$, proanalysis grade, Taurus) and subsequently distilled and stored over freshly activated molecular sieves (4 Å, Aldrich). Argon (Ar, 99.99\%, Air Liquide) and oxygen $\left(\mathrm{O}_{2}, 99 \%\right.$, Air Liquide $)$ were used as received.

All employed modified dyes were kindly provided by Professor F. Amat-Guerri (Instituto de Química Orgánica, CSIC, Madrid, Spain). RB (95\%), EoY ( 99\%), and EoB (95\%) were purchased from Aldrich. Both the commercial 
<smiles>CO[Si](CCCOCC1CO1)(OC)OC</smiles>

DA

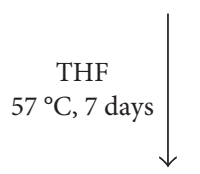

GPTMS

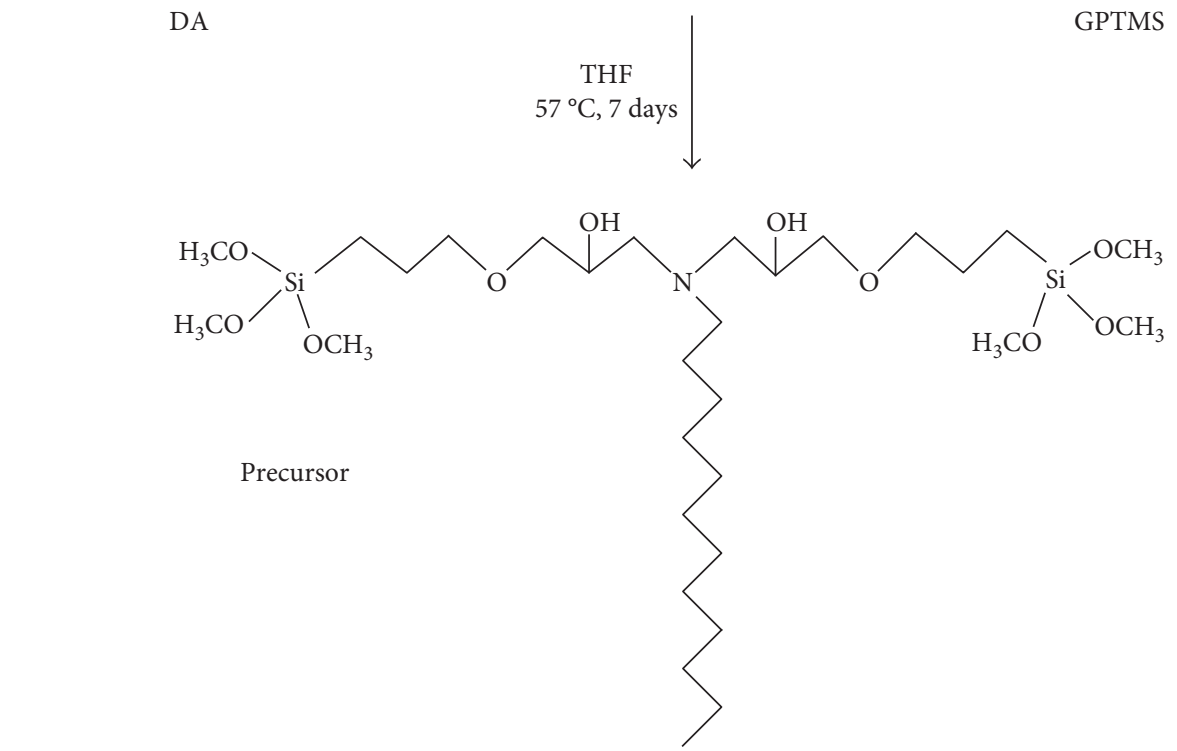

Hydrolysis
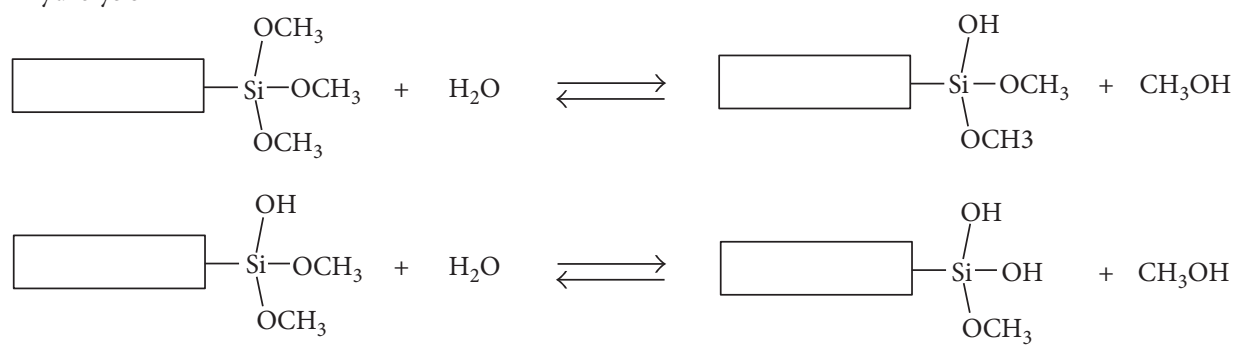

Condensation

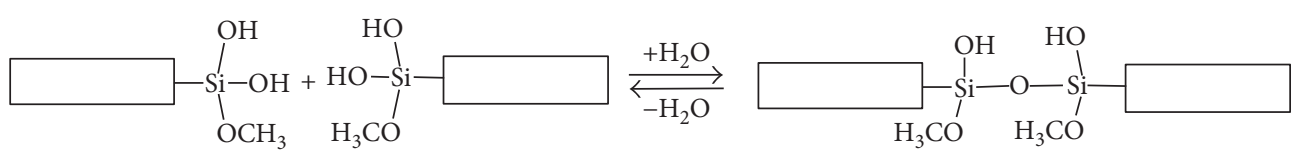

(a)
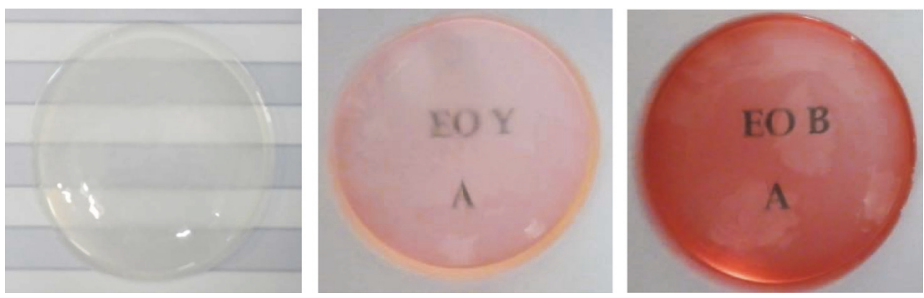

(b)

FIGURE 2: (a) Schematic representation of the precursor synthesis, its hydrolysis, and (partial) condensation to produce the corresponding silsesquioxanes. (b) Photographs of the neat and dye-doped SSO films.

dyes and their derivatives were rigorously purified by preparative chromatographic methods and subsequently recrystallized. EoB was recrystallized from methanol. Details of purification methods can be found in reference [28].

2.2. Synthesis of Silsesquioxane Films. Silsesquioxane films were obtained following the method reported by Gómez et al. [12]. Figure 2(a) shows the film preparation procedure. Briefly, the precursor was synthesized by mixing stoichiometric amounts of DA:GPTMS $(1: 2) 0.4 \mathrm{M}$ and $0.8 \mathrm{M}$, respectively, prepared in THF. The reaction mixture was heated at $57^{\circ} \mathrm{C}$ for 7 days until complete conversion was reached. The hydrolysis and condensation reactions were carried out from a solution $0.1 \mathrm{M}$ of the precursor in $\mathrm{THF}$ 
and the appropriate amounts of water and catalyst, so that the final molar ratio in the reaction mixture was as follows: $\mathrm{Si}: \mathrm{H}_{2} \mathrm{O}: \mathrm{HCOOH}=1: 3: 0.1$. The solution was stirred vigorously and then poured into Delrin ${ }^{\circledR}$ plates $(d=5 \mathrm{~cm})$ until the liquid reached $\sim 5 \mathrm{~mm}$ high. The plates were covered with aluminum foil and placed in a convection oven at $30^{\circ} \mathrm{C}$ for $24 \mathrm{~h}$. After evaporation of the solvent, films of approximately 500 microns thick were obtained by casting. The films were quite transparent, flexible, and easily detachable from the plates. Dyes were incorporated into the silsesquioxane matrix before the hydrolysis/condensation processes. The concentrations of the different dyes were adjusted so that their absorbance in the films were $\sim 0.1$ and $\sim 0.8$ for the emission and transient absorption experiments, respectively. A low refraction index of 1.50 approximately is observed. A photograph of the transparent films is presented in Figure 2(b).

It is important to note that no dye leaching was observed when doped matrices were exposed to different solvents (water, THF, and ACN) for about one week. Moreover, the dye-doped films maintained their color and photosensitizing properties after being stored for two years at laboratory conditions.

2.3. Instrumental. Absorption spectra of films were obtained with a Shimadzu UV-2401 PC spectrophotometer using an integrating sphere module (model ISR 2200) to minimize scattering effects. Emission spectra were recorded using a Horiba Jobin Yvon Fluoromax 4 fluorimeter.

Fluorescence lifetime measurements were performed with an Edinburgh Instruments OB 900 time-correlated single-photon counting fluorimeter. The excitation of the samples was carried out with a PicoQuant PLS450 diode with emission centered at $\sim 450 \mathrm{~nm}$ and pulse FWHM of $0.8 \mathrm{~ns}$. The emission wavelengths were selected near the maximum of fluorescence maxima of the dyes in each medium. In all cases, the fluorescence lifetimes could be well fitted to monoexponential (or biexponential) decay functions.

Transient absorption spectra were recorded using a home-made laser flash photolysis equipment. A Spectron SL400 Nd:YAG laser (532 nm, 18 ns pulse width) was used as an excitation source. The laser beam was defocused to cover all the path length $(10 \mathrm{~mm})$ of the analyzing beam from a $150 \mathrm{~W}$ Xe lamp. The detection system comprised a monochromator (Photon Technology International) coupled to a photomultiplier tube (Hamamatsu R666). The signal, acquired by a digitizing scope (Hewlett-Packard 54504), was averaged and then transferred to a computer. Solutions were purged with argon to reduce molecular oxygen concentration. Films were fixed to a glass support and placed in a front surface with respect to the excitation laser beam.

Photooxidation assays of hydrocarbons were conducted by selectively irradiating the dyes with two green LEDs $\left(\lambda_{\text {center-emission }} \sim 510 \mathrm{~nm}, \quad\right.$ FWHM $\sim 45 \mathrm{~nm}$, optical power $\sim 6 \mathrm{~mW}$ per LED) and simultaneously monitoring the decrease in absorbance of DMA as function of time. Absorption spectra were automatically collected at constant time intervals using a UV-Vis spectrophotometer (HP 8452A, Hewlett-Packard) equipped with a kinetic software module.
Kinetic traces were constructed by plotting the absorption at indicated wavelengths (corresponding to absorption maxima of DMA) as a function of LED irradiation time and correcting these values by variations in lamp intensity over time as monitored in a region where the sample does not absorb (750-800 nm). Special care was taken to avoid obstruction of the spectrophotometer optical path by the film.

\section{Results and Discussion}

3.1. Photophysical Characterization. The absorption $\left(\lambda_{A}^{\max }\right)$ and emission maxima $\left(\lambda_{E}^{\max }\right)$ of Eo, RB, and its derivatives measured in ACN, DMSO, and THF and in the SSO films are summarized in Table 1 . The results shown that the absorption and emission maxima of the dyes incorporated into the SSO films are similar to those observed in fluid media. No differences were observed for the excitation spectra of the dyes in the solution or those incorporated into the SSO film, with respect to their correspondent absorption spectra.

Figure 3 shows the absorption and emission spectra of the neat SSO film and RB and EoY in the SSO films. Emission spectra were taken with excitation at 520, 490, and $350 \mathrm{~nm}$ for RB, EoY, and neat SSO, respectively.

The emission spectra of all hybrid materials doped with the xanthenic dyes are shown in Figure 4. As can be seen in the figure, the emission spectra are consistent with those of the dyes in the solution. This indicates that the photophysical properties of the dyes remain unchanged when incorporated into the SSO matrix. The Stokes shifts calculated for the dyes in THF are very similar to those calculated in the SSO matrices (Table 1) suggesting analogous microenvironment interactions in both media.

As reported previously, the SSO matrix displays an emission band between 300 and $400 \mathrm{~nm}$, in which maximum depends on the excitation wavelength [29]. As shown in Figure 4, the emission of the dye-doped films is bathochromically shifted relative to that of the neat films. The presence of the dyes in the films allows excitation with light of lower energies and to obtain emission at longer wavelengths.

Fluorescence lifetimes $(\tau)$ were measured for EoY, EoB, $\mathrm{RB}$, and its derivatives in THF and in SSO films and presented in Table 2. The emission decay profiles in THF were clearly monoexponential. However, in the SSO matrixes, the emission decays are multiexponential. Figure 5 shows the exponential decays of EoY in THF (Figure 5(a)) and in the SSO matrix (Figure 5(b)). The profiles can be acceptably fitted to biexponential decays (Table 2) or, better yet, to lifetime distributions using a specialized maximum entropy method [30]. In any case, results indicate that in the SSO matrices, the emission decays are always bimodal. Interestingly, SSO-EoY and SSO-EoB films show a fast decay with small amplitude and a dominant slower decay $(\sim 85 \%)$ with a lifetime matching that of the dye dissolved in THF. In contrast, none of the estimated $\tau$ for RB in the film agrees with that observed in THF. As a common behavior, two lifetimes are observed when the dyes (commercials or synthetics) were incorporated into the SSO films. Similar behaviors have been reported for other organic fluorophores confined into rigid matrices [30]. 
TABLE 1: Absorption $\left(\lambda_{A}^{\max }\right)$ and emission maxima $\left(\lambda_{E}^{\max }\right)$ of Eo, RB, and its derivatives measured in ACN, DMSO, and THF and in the SSO films. The corresponding Stokes shifts are also listed.

\begin{tabular}{lccc}
\hline Sample & $\lambda_{\mathrm{abs}}(\mathrm{nm})$ & $\lambda_{\mathrm{em}}(\mathrm{nm})$ & Stokes shift $\left(\mathrm{cm}^{-1}\right)$ \\
\hline RB-ACN & 559 & 570 & 345 \\
RB-DMSO & 565 & 576 & 338 \\
RB-THF & 561 & 588 & 818 \\
\hline EoY-ACN & 529 & 545 & 555 \\
EoY-DMSO & 544 & 561 & 557 \\
EoY-THF & 533 & 557 & 808 \\
\hline EoB-ACN & 527 & 585 & 1881 \\
EoB-DMSO & 545 & 597 & 1598 \\
EoB-THF & 527 & 570 & 1431 \\
\hline SSO & 300 & 431 & 10,131 \\
\hline SSO-RB & 565 & 586 & 634 \\
SSO-RB3 & 567 & 584 & 513 \\
\hline SSO-EoY & 534 & 555 & 708 \\
SSO-EoB & 534 & 618 & 2545 \\
SSO-Eo2 & 528 & 553 & 856 \\
SSO-Eo3 & 543 & 564 & 686 \\
\hline
\end{tabular}

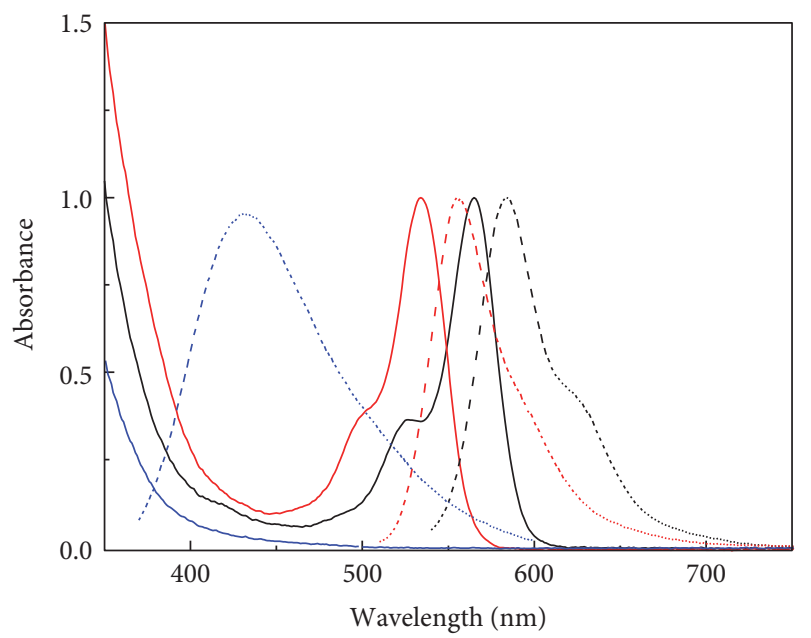

Figure 3: Normalized absorption (solid line) and emission (dashed line) spectra of RB (red) and EoY (black) in the SSO films and of the neat SSO matrix (blue).

The previous observations can be rationalized considering that fluorophores dispersed in the microheterogeneous materials are able to sense environments with different polarities, viscosities, $\mathrm{H}$-bonding capabilities, and so forth. In most cases, confinement affects the rates of the nonradiative relaxation processes (i.e., intersystem crossing and internal conversion) of the excited state by affecting (hindering or enabling) different vibrational modes coupled to the deactivation pathways [30]. Hence, confinement effects depend on the nature of the fluorophore and the matrix, and they cannot be easily anticipated.

To complete the photophysical characterization of the synthesized materials, laser flash photolysis studies were

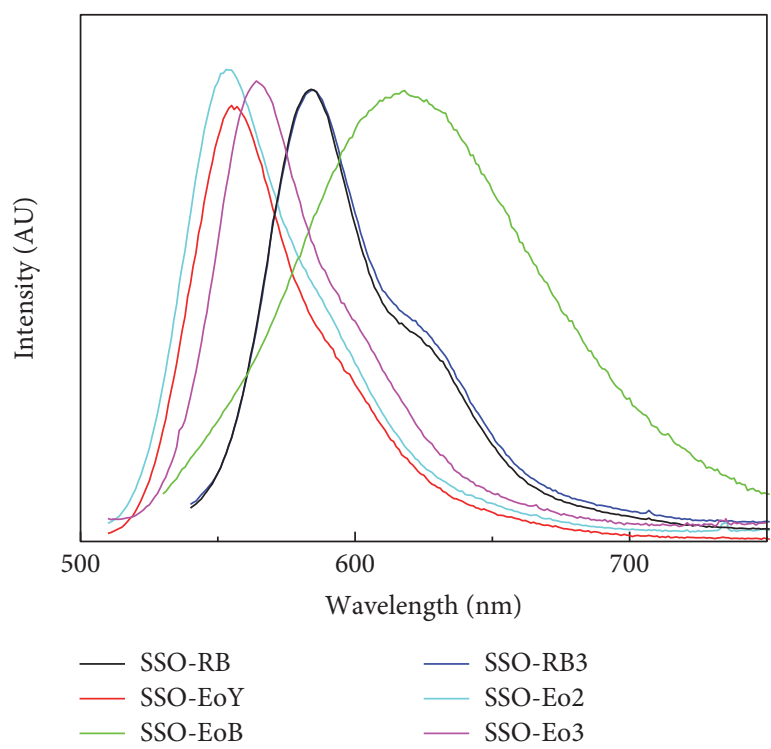

FIGURE 4: Emission spectra of SSO doped with xanthenic dyes.

TABLE 2: Fluorescence lifetimes $(\tau)$ measured for Eo, RB, and its derivatives in THF and in the SSO films.

\begin{tabular}{lccc}
\hline Sample & $\tau 1(\mathrm{~ns}) \%$ & $\tau 2(\mathrm{~ns}) \%$ & $\chi^{2}$ \\
\hline RB-THF & $2.63(100 \%)$ & - & 1.170 \\
\hline EoY-THF & $3.91(100 \%)$ & - & 1.016 \\
\hline EoB-THF & $3.63(100 \%)$ & - & 1.064 \\
\hline SSO-RB & $1.97(79 \%)$ & $6.80(21 \%)$ & 1.044 \\
SSO-EoY & $0.26(14 \%)$ & $3.90(86 \%)$ & 1.007 \\
SSO-EoB & $0.84(16 \%)$ & $3.68(84 \%)$ & 1.063 \\
SSO-Eo2 & $0.07(5 \%)$ & $3.88(95 \%)$ & 1.038 \\
SSO-Eo3 & $0.16(22 \%)$ & $4.09(78 \%)$ & 1.239 \\
SSO-RB3 & $2.01(80 \%)$ & $7.02(20 \%)$ & 1.081 \\
\hline
\end{tabular}

carried out to determine the transient absorption spectra of the dyes incorporated into the SSO matrix. The results showed that the transient absorption spectra of the dyes in the matrix correspond to those reported in literature for the dyes in the solution $[31,32]$. As an example, in Figure 6, the transient absorption spectra of EoY in THF and in the SSO film and Eo2 in SSO are displayed.

These transient absorption spectra present two characteristic bands in the regions of $350-460$ and $550-700 \mathrm{~nm}$ that are assigned to the triplet state of the dyes. The negative values of $\Delta \mathrm{Abs}$ correspond to bleaching of the dye ground state absorptions. Similar spectra were obtained for all dyes.

In all cases, the transient spectra obtained in the films match with those obtained in the solution, which allows to conclude that dye triplet excited states are formed independently of the properties of the medium (rigidity, polarity, etc.).

3.2. Photooxidation of DMA in the Presence of the SSO Films. Photooxidation of DMA in the presence of doped films were 

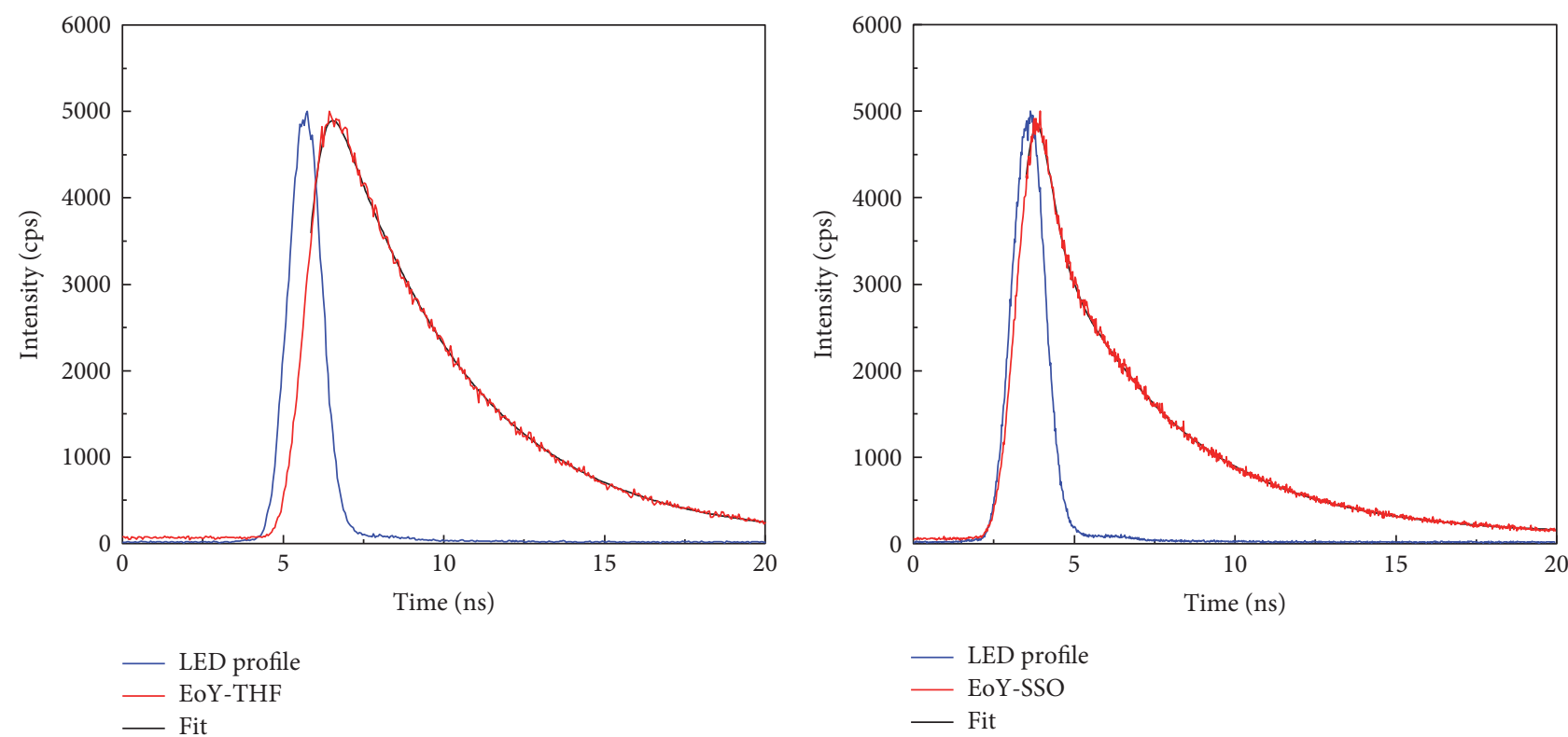

(a)

(b)

FIGURE 5: Emission decay of EoY in (a) THF solution and in (b) the solid SSO film.

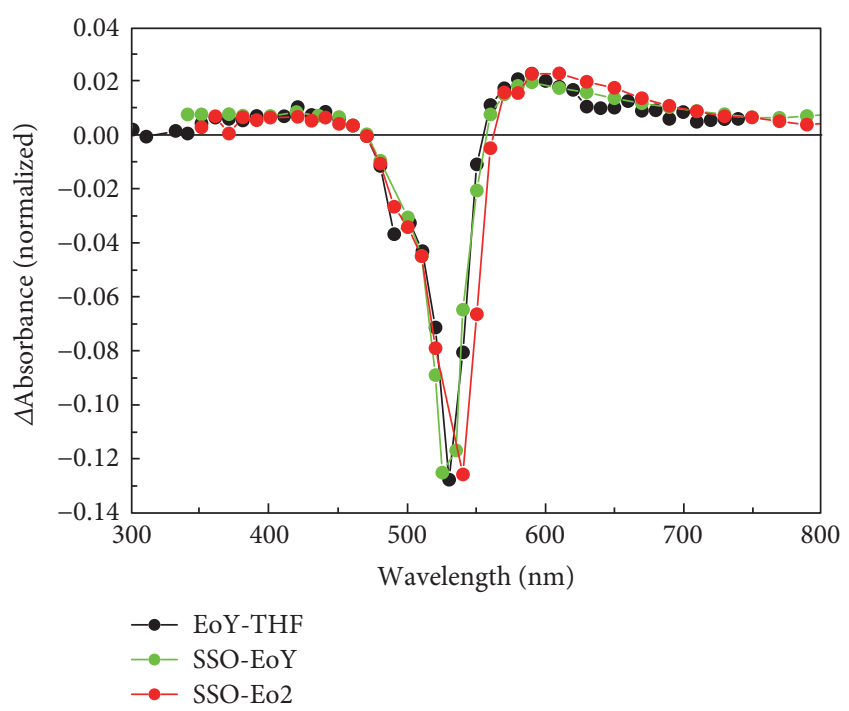

Figure 6: Normalized transient absorption spectrum of EoY in deaerated THF and of EoY and Eo2 in the SSO films, extrapolated at zero time.

performed in ACN. DMA exhibits high reactivity towards singlet oxygen forming a stable endoperoxide derivative $\left(\right.$ DMA-O ${ }_{2}$ ) via a Diels-Alder cycloaddition across its middle ring. The concentration of DMA in ACN solutions containing the films doped with different xanthenic dyes was observed to decrease upon selective film irradiation with a green LEDs under air-equilibrated conditions. The disappearance of DMA is followed by a monitored absorbance decrease at $398 \mathrm{~nm}$ (the endoperoxide does not absorb at $398 \mathrm{~nm}$ ). The results are summarized in Figure 7.

As shown in Figure 7, upon film irradiation, a significant decrease in DMA concentration can be observed for the SSO-

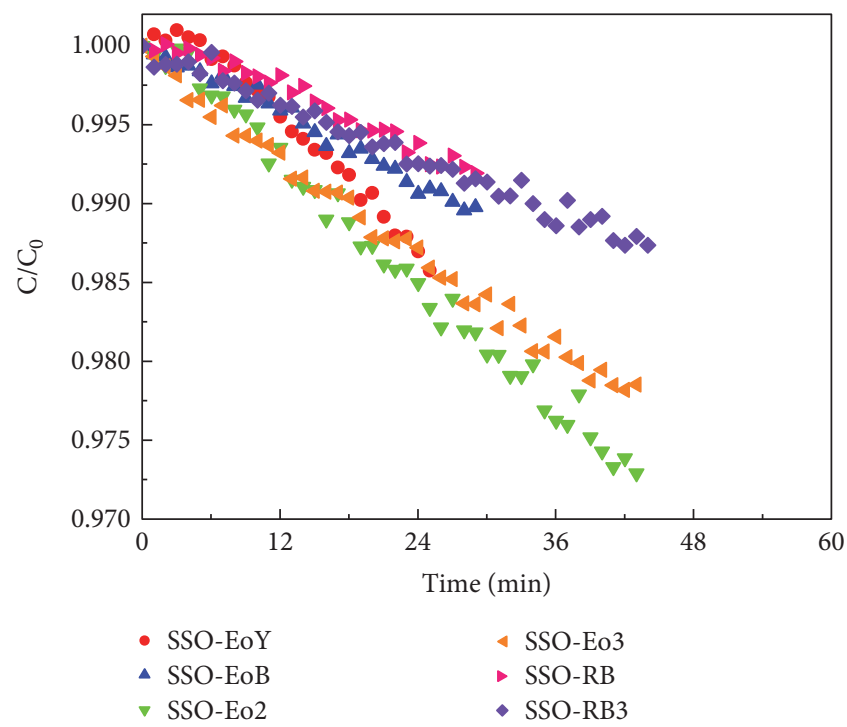

Figure 7: Oxidation of DMA in ACN $\left(\sim 8 \times 10^{-5} \mathrm{M}\right)$ solutions sensitized by photoirradiation of the SSO films doped with xanthenic dyes in the presence of two green LEDs.

doped samples, which is associated with the photosensitized formation of $\mathrm{O}_{2}\left({ }^{1} \Delta_{\mathrm{g}}\right)$ and the subsequent reaction of DMA with $\mathrm{O}_{2}\left({ }^{1} \Delta_{\mathrm{g}}\right)$ to yield DMA-O ${ }_{2}$. DMA consumption was not observed in a control sample without a film indicating that autophotosensitization of DMA is insignificant under the employed conditions.

The above results are consistent with the significant overlap of the emission spectrum of the green LEDs with the absorption spectra of the doped SSO film and with the spectral mismatch between LED emission and DMA absorption spectra. These results confirm the ability of the xanthenic 
dyes incorporated in the SSO films to photosensitize the formation of $\mathrm{O}_{2}\left({ }^{1} \Delta_{\mathrm{g}}\right)$ under green LED irradiation.

\section{Conclusions}

In this work, hybrid materials based on silsesquioxanes with embedded xanthenic dyes as dopants were synthesized. The novel polymeric materials are thermally and chemically stable, optically homogeneous, and translucid and have low refractive index.

Because the dyes used maintain their photophysical properties in the polymer matrix of SSO for years, these doped hybrid materials could be used as decontaminants of recreational and drinking waters, since the dyes included in the matrix induce the oxidation of organic pollutants through the photosensitized generation of singlet oxygen. Moreover, these materials have the advantage of being easily removed from the treated fluid after their application, in contrast to nano-/microparticulate systems, offering an additional advantage for their application.

\section{Disclosure}

The funders had no role in the study design, data collection and analysis, decision to publish, or preparation of the manuscript.

\section{Conflicts of Interest}

The authors declare that they have no competing interests. María Lorena Gómez, Rodrigo E. Palacios, Carlos A. Chesta, and Hernán A. Montejano are permanent research staff of CONICET.

\section{Acknowledgments}

The authors acknowledge financial support for this work by grants from ANPCyT, Argentina (PICT 1439/2013); CONICET, Argentina (PIP 11220150100295/2015); and Secretaría de Ciencia y Técnica (SECyT), UNRC (PPI 2016), Argentina. Carolina V. Waiman thanks CONICET for postdoctoral scholarships.

\section{References}

[1] B. Petrie, R. Barden, and B. Kasprzyk-Hordern, "A review on emerging contaminants in wastewaters and the environment: current knowledge, understudied areas and recommendations for future monitoring," Water Research, vol. 72, pp. 3-27, 2015.

[2] D. L. Sparks, Environmental Soil Chemistry, Academic Press, New York, 2nd edition, 2003.

[3] J. Herney-Ramirez, M. A. Vicente, and L. M. Madeira, "Heterogeneous photo-Fenton oxidation with pillared claybased catalysts for wastewater treatment: a review," Applied Catalysis B: Environmental, vol. 98, pp. 10-26, 2010.

[4] S. Vilhunen and M. Sillanpää, "Recent developments in photochemical and chemical AOPs in water treatment: a mini-review," Environmental Science and Bio/Technology, vol. 9, pp. 323-330, 2010.
[5] S. Singh, H. Mahalingam, and P. K. Singh, "Polymer-supported titanium dioxide photocatalysts for environmental remediation: a review," Applied Catalysis A: General, vol. 462-463, pp. 178-195, 2013.

[6] T. A. Dahl, W. R. Midde, and P. E. Hartman, "Pure singlet oxygen cytotoxicity for bacteria," Photochemistry and Photobiology, vol. 46, no. 3, pp. 345-352, 1987.

[7] A. J. Acher, E. Fischer, R. Zellingher, and Y. Manor, "Photochemical disinfection of effluents - pilot plant studies," Water Research, vol. 24, no. 7, pp. 837-843, 1990.

[8] M. M. Khin, A. S. Nair, V. J. Babu, R. Murugan, and S. Ramakrishna, "A review on nanomaterials for environmental remediation," Energy \& Environmental Science, vol. 5, pp. 8075-8109, 2012.

[9] C. V. Waiman, I. E. dell'Erba, C. A. Chesta, and M. L. Gómez, "Hybrid films based on a bridged silsesquioxane doped with goethite and montmorillonite nanoparticles as sorbents of wastewater contaminants," Journal of Nanomaterials, vol. 2016, Article ID 6286247, 9 pages, 2016.

[10] E. W. de Menezes, E. C. Lima, B. Royer et al., "Ionic silica based hybrid material containing the pyridinium group used as an adsorbent for textile dye," Journal of Colloid and Interface Science, vol. 378, pp. 10-20, 2012.

[11] H. B. He, B. Li, J. P. Dong et al., "Mesostructured nanomagnetic polyhedral oligomeric silsesquioxanes (POSS) incorporated with dithiol organic anchors for multiple pollutants capturing in wastewater," ACS Applied Materials \& Interfaces, vol. 5, pp. 8058-8066, 2013.

[12] M. L. Gómez, C. E. Hoppe, I. A. Zucchi, R. J. J. Williams, M. I. Giannotti, and M. A. López-Quintela, "Hierarchical assemblies of gold nanoparticles at the surface of a film formed by a bridged silsesquioxane containing pendant dodecyl chains," Langmuir, vol. 25, pp. 1210-1217, 2009.

[13] M. L. Gómez, D. P. Fasce, R. J. J. Williams, C. M. Previtali, and H. A. Montejano, "Transparent polysilsesquioxane films obtained from bridged ureasil precursors: tunable photoluminescence emission in the visible region and filtering of UV-radiation," Macromolecular Materials and Engineering, vol. 295, pp. 1042-1048, 2010.

[14] M. L. Gómez, C. E. Hoppe, and R. J. J. Williams, "In situ generation of silver microstructures by thermal decomposition of silver $\mathrm{n}$-dodecanethiolate dispersed in an organic-inorganic hybrid coating," Materials Chemistry and Physics, vol. 130, no. 1, pp. 519-523, 2011.

[15] M. G. Alvarez, M. L. Gómez, S. J. Mora, M. E. Milanesio, and E. N. Durantini, "Photodynamic inactivation of Candida albicans using bridged polysilsesquioxane films doped with porphyrin," Bioorganic \& Medicinal Chemistry, vol. 20, no. 13, pp. 4032-4039, 2012.

[16] R. M. Spada, M. Cepeda-Plaza, M. L. Gomez et al., "Clean singlet oxygen production by a $\mathrm{Re}(\mathrm{I})$ complex embedded in a flexible self-standing polymeric silsesquioxane film," The Journal of Physical Chemistry C, vol. 119, pp. 10148-10159, 2015.

[17] A. A. Gorman and M. A. J. Rodgers, "Singlet molecular oxygen," Chemical Society Reviews, vol. 10, pp. 205-231, 1981.

[18] A. P. Schaap, A. L. Thayer, E. C. Blossey, and D. C. Neckers, "Polymer-based sensitizers for photooxidations. II," Journal of the American Chemical Society, vol. 97, no. 13, pp. 37413745, 1975.

[19] D. Bürget, J. P. Fouassier, F. Amat-Gerri, R. Mallavia, and R. Sastre, "Enhanced activity as polymerization photoinitiators 
of rose bengal and eosin esters with an O-benzoyl- $\alpha$-oxooxime group: the role of the excited state reactivity," Acta Polymerica, vol. 50, no. 9, pp. 337-346, 1999.

[20] F. Amat-Guerri, M. M. C. López-González, R. Sastre, and R. Martínez-Utrilla, "Spectrophotometric determination of ionization and isomerization constants of rose bengal, eosin Y and some derivatives," Dyes and Pigments, vol. 13, no. 3, pp. 219-232, 1990.

[21] M. V. Encinas, A. M. Rufs, S. G. Bertolotti, and C. M. Previtali, "Xanthene dyes/amine as photoinitiators of radical polymerization: a comparative and photochemical study in aqueous medium," Polymer, vol. 50, pp. 2762-2767, 2009.

[22] N. A. García and F. Amat-Guerri, "Photodegradation of hydroxylated $\mathrm{N}$-heteroaromatic derivatives in natural-like aquatic environments. A review of kinetic data of pesticide model compounds," Chemosphere, vol. 59, pp. 1067-1082, 2005.

[23] W. J. Jones, A. Grofcsik, M. Kubinyi, and D. Thomas, "Concentration-modulated absorption spectroscopy and the triplet state: photoinduced absorption/bleaching in erythrosin B, rose bengal and eosin y," Journal of Molecular Structure, vol. 792, pp. 121-129, 2006.

[24] D. Costley, H. Nesbitt, N. Ternan et al., "Sonodynamic inactivation of gram-positive and gram-negative bacteria using a rose bengal-antimicrobial peptide conjugate," International Journal of Antimicrobial Agents, vol. 49, no. 1, pp. 31-36, 2017.

[25] L.-L. He, X.-X. Wu, Y.-X. Wang et al., "Spectroscopic investigation on the sonodynamic damage to protein in the presence of eosine B," Ultrasonics Sonochemistry, vol. 26, pp. 93-98, 2015.

[26] M.-A. H. Al-Akhras, K. Aljarrah, B. Albiss, and D. Al-Khalili, "Influence of iron oxide nanoparticles (Fe3O4) on erythrocyte photohemolysis via photofrin and rose bengal sensitization," Photodiagnosis and Photodynamic Therapy, vol. 18, pp. 111118, 2017.

[27] R. Schmidt, K. Schaffner, W. Trost, and H. D. Brauer, "Wavelength-dependent and dual photochemistry of the endoperoxides of anthracene and 9,10-dimethylanthracene," The Journal of Physical Chemistry, vol. 88, no. 5, pp. 956-958, 1984.

[28] F. Amat-Guerri, M. M. C. Lopez-Gonzalez, R. MartinezUtrilla, and R. Sastre, "Synthesis and spectroscopic properties of new rose bengal and eosin Y derivatives," Dyes and Pigments, vol. 12, no. 4, pp. 249-272, 1990.

[29] M. L. Gómez, I. E. dell' Erba, C. A. Chesta, C. E. Hoppe, and R. J. J. Williams, "Dispersion of gold dodecanethiolate in a silsesquioxane film with pendant dodecyl chains: from photoluminescent materials to gold nanocomposites," Journal of Materials Science, vol. 48, pp. 8559-8565, 2013.

[30] C. Solis, J. J. Torres, N. Gsponer et al., "Energy and electron transfer processes in polymeric nanoparticles," Photochemical \& Photobiological Sciences, vol. 12, pp. 2146-2159, 2013.

[31] D. Burget and J. P. Fouassier, "Laser flash photolysis studies of the interaction of rose bengal with an iron arene complex," Journal of the Chemical Society - Faraday Transactions, vol. 94, no. 13, pp. 1849-1854, 1998.

[32] S. D. M. Islam, T. Konishi, M. Fujitsuka, O. Ito, Y. Nakamura, and Y. Usui, "Photosensitized reduction of methyl viologen using eosin- $\mathrm{Y}$ in presence of a sacrificial electron donor in water-alcohol mixture," Photochemistry and Photobiology, vol. 71, no. 6, pp. 675-680, 2000. 

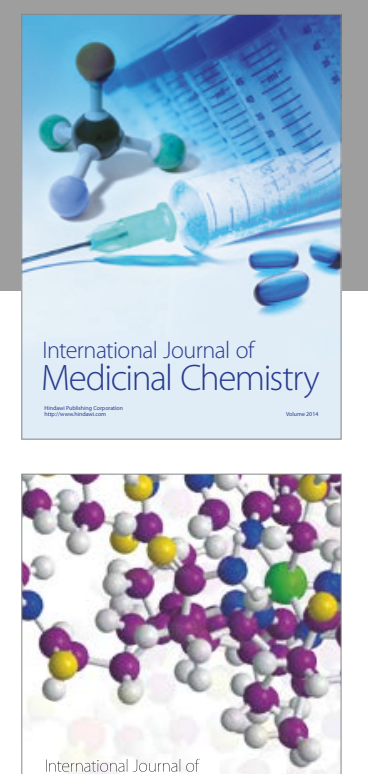

Carbohydrate Chemistry

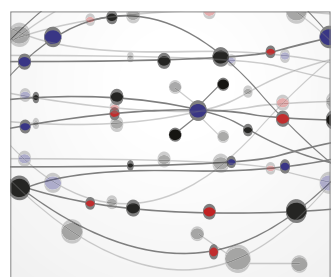

The Scientific World Journal
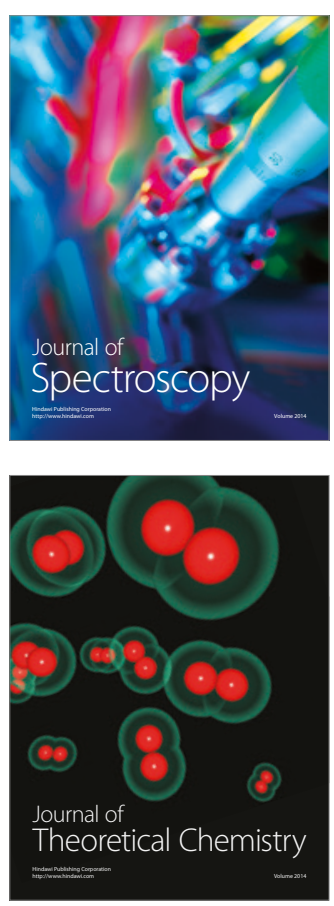
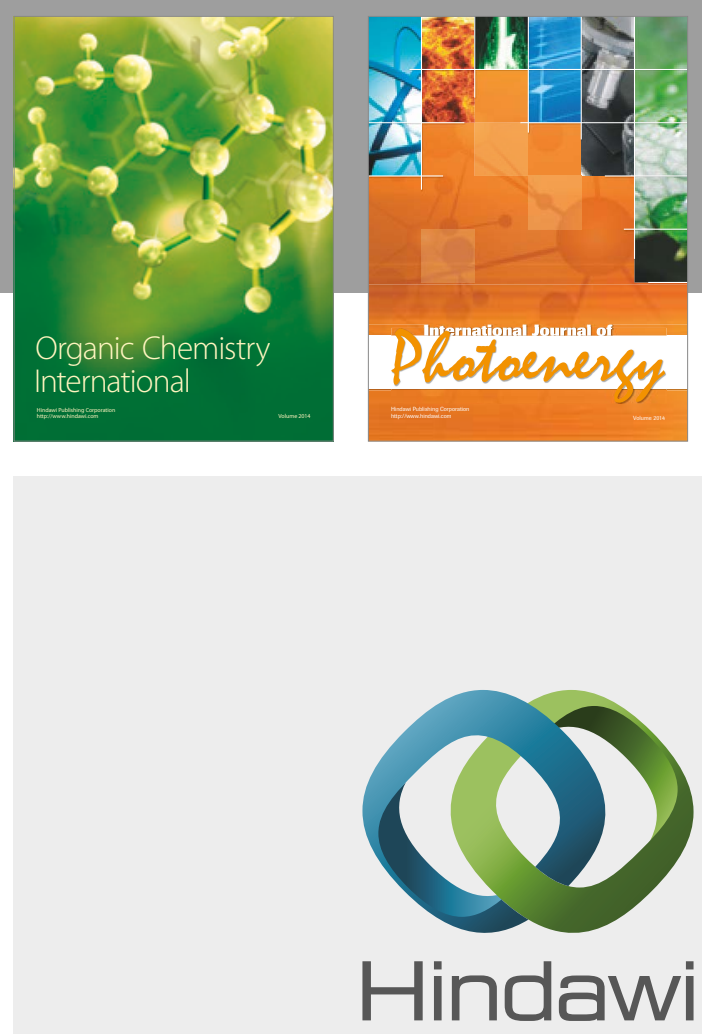

Submit your manuscripts at

https://www.hindawi.com

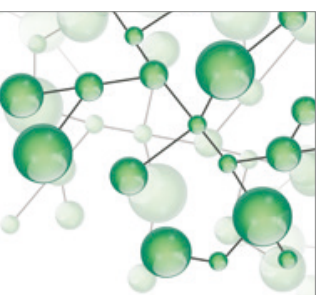

International Journal of

Inorganic Chemistry

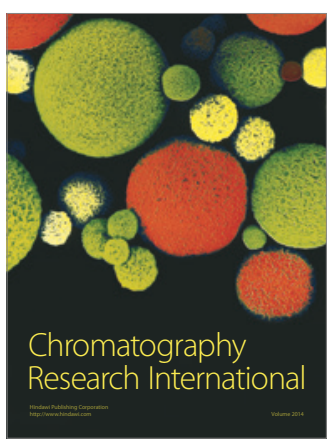

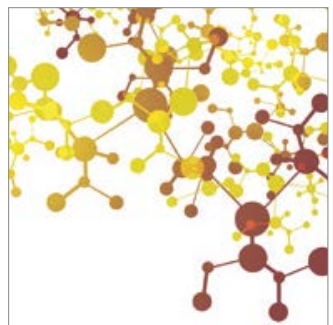

Applied Chemistry
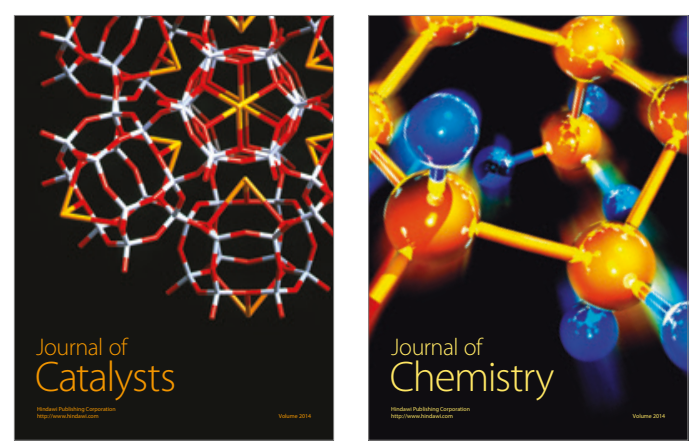
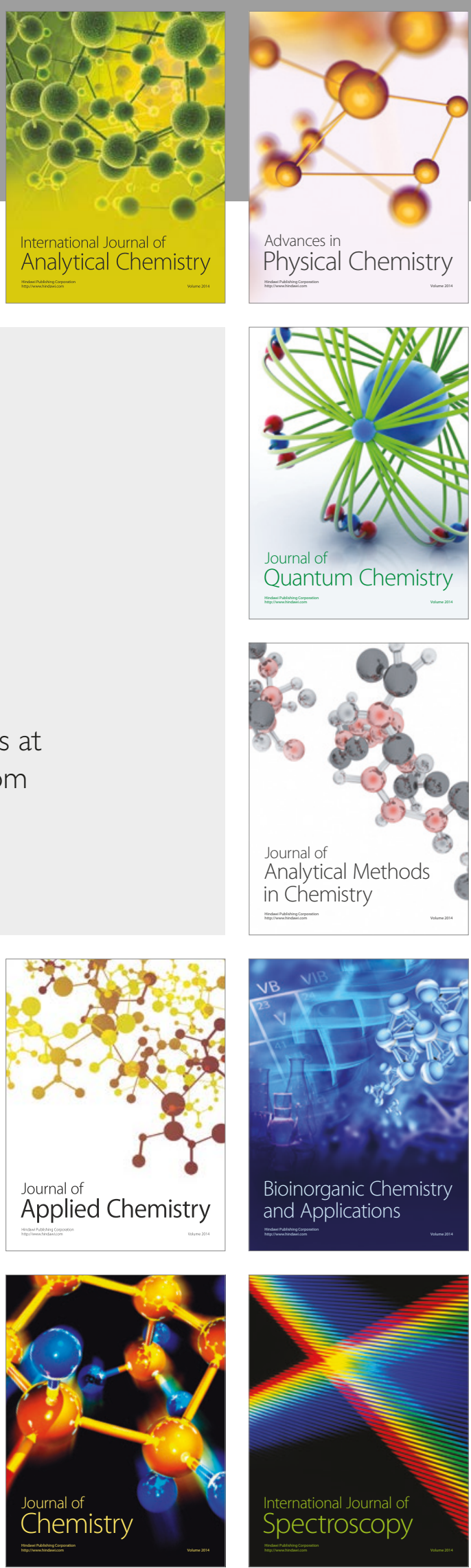